Bull. Mater. Sci., Vol. 8, No. 2, May 1986, pp. 183-191. (C) Printed in India.

\title{
Computer-aided roll pass design for continuous billet mill
}

\author{
ASHOK KUMAR, SUDHAKER JHA and V RAMASWAMY \\ Research \& Development Centre for Iron and Steel, Steel Authority of India Limited, Ranchi, \\ India
}

\begin{abstract}
Ahstract. A mathematical model has been developed for computing the geometrical dimensions of square-diamond square pass sequence for a continuous billet mill. The model is based on derivation of shape and size factor from the geometry of the pass taking into account pass filling, pass rounding etc. Using these factors and a basic equation of spread for flat rolling, a governing equation incorporating angle of diamond and reduction in consecutive passes has been formulated. Newton's substitution method has been used to solve the equation. With known reduction between consecutive passes, geometrical dimension of square and diamond passes are computed. A model has been used to calculate pass design of a finishing train of a continuous billet mill producing $60 \mathrm{~mm}$ square billet from $120 \mathrm{~mm}$ square bloom. The elongation values have been optimized by varying the apex angle of diamond. A close agreement between computed and actual values shows the validity of the model.
\end{abstract}

Keywords. Geometrical dimensions; square-diamond pass; computer-aided model; pass sequence; apex angle; rolling force; torque

\section{Introduction}

Diamond-square pass sequence is used in the finishing group of stands of a continuous billet mill to produce round-cornered squares. Conventionally the sequence is designed (Burtsev 1973) by obtaining elongation coefficient on the principle of having width of any given pass equal to the height of the preceding pass. Form changes in diamond passes have also been studied experimentally (Zaitsev and Anufriev 1969) and nomograms have been prepared for obtaining pass dimensions. However, these methods generally require trial and error to establish roll pass design and at the same time do not effectively utilise available biting ability, mill power etc of different mill stands. To overcome these shortcomings, a software package was developed at this centre for computer-aided pass design for continuous billet mill. This paper outlines the approach adopted and compares the results obtained from the computer model developed with the actual mill performance.

\section{Approach}

Diamond-square pass sequence can be fully developed if the elongation coefficient $(\lambda)$ between two consecutive passes is known. In the present work the coefficient is obtained by converting the diamond square passes into equivalent rectangles having width equal to their effective width and the Ekelund spread formula (Jha 1980) has been modified and made use of. This offers the advantage of calculating reduction sequence starting from the last finishing pass and is superior in accuracy to other spread formulae

A list of symbols appears at the end of the paper. 
(Jha 1980). With the calculated value of $\lambda$, for an assumed angle of diamond, the dimension of pass in the next mill stand is obtained. Limitations of permissible angle of bite, mill speed, power force parameters etc have been observed. If needed a change in apex angle of diamond pass is effected and this in turn changes the value of $\lambda$ and pass dimensions. Computation is repeated for all the mill stands and the dimensions of ingoing bloom entering the finishing mill are figured. If there is a limitation on bloom size as well, $\lambda$ is readjusted again by changing the apex angle of diamond in passes of different mill stands.

\subsection{Elongation coefficient}

Figure 1 shows a diamond bar going into square pass along with their equivalent rectangles. If

$$
\delta=b_{1} / h_{1} ; n=h_{2} / b_{2} \text { and } \gamma=h_{2} / h_{1} .
$$

Elongation coefficients $(\lambda)$ for the pair of pass will be

$$
\lambda=A_{1} / A_{2}=b_{1} h_{1} / b_{2} h_{2}=n \cdot \delta / \gamma^{2} \text {. }
$$

The modified Ekelund spread equation is given as

$$
\beta=1+\frac{2(1-\gamma)}{1+\gamma} \cdot \frac{M}{M+1},
$$

where $\quad M=\frac{0.8}{\left(b_{1} / l d\right)^{2}}\left[2-\frac{3\left(h_{1}-h_{2}\right)}{l d}\right]$

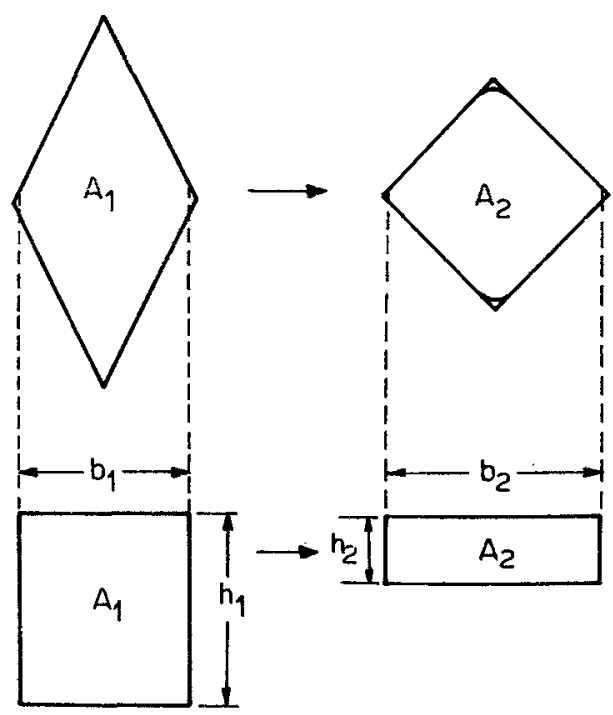

Figure 1. Diamond and square passes with their equivalent rectangles. 
The final equation can be written as

$$
n=\frac{(\gamma / \delta)}{1+\frac{2(1-\gamma)}{1+\gamma}\left[\frac{0.4 \gamma(1-\gamma)\left\{2-3\left[\left(\frac{2(1-\gamma)}{\gamma}\right)\left(\frac{h_{2}}{D}\right)\right]^{1 / 2}\right\}}{\delta^{2} \frac{h_{2}}{D}+0.4 \gamma(1-\gamma)\left\{2-3\left[\left(\frac{2(1-\gamma)}{\gamma}\right)\left(\frac{h_{2}}{D}\right)\right]^{1 / 2}\right\}}\right]}
$$

Equations (1) and (2) can be used for determining elongation coefficients $(\lambda)$ if $\delta, n$ and $h 2 / D$ are known.

\subsection{Shape factors}

$\delta$ and $n$ largely depend on the geometrical shape of passes and are therefore termed shape factors. For any pass, $\delta \cdot n=1$. Considering pass filling and apex rounding of diamond and square passes, the shape and size factor $\left(h_{2} / D\right)$ can be written as shown in figure 2.

\subsection{Rolling force and torque}

Roll separating force and rolling torque were computed using the following equations, which are derived from the basic theory of deformation for rolling of square and diamond sections (Jha et al 1983).

\subsection{Roll force}

$$
F=\sigma \cdot l d \cdot n_{\sigma}\left[\frac{2 W}{3}-\frac{H_{1} \cdot W}{10 R}\right]
$$

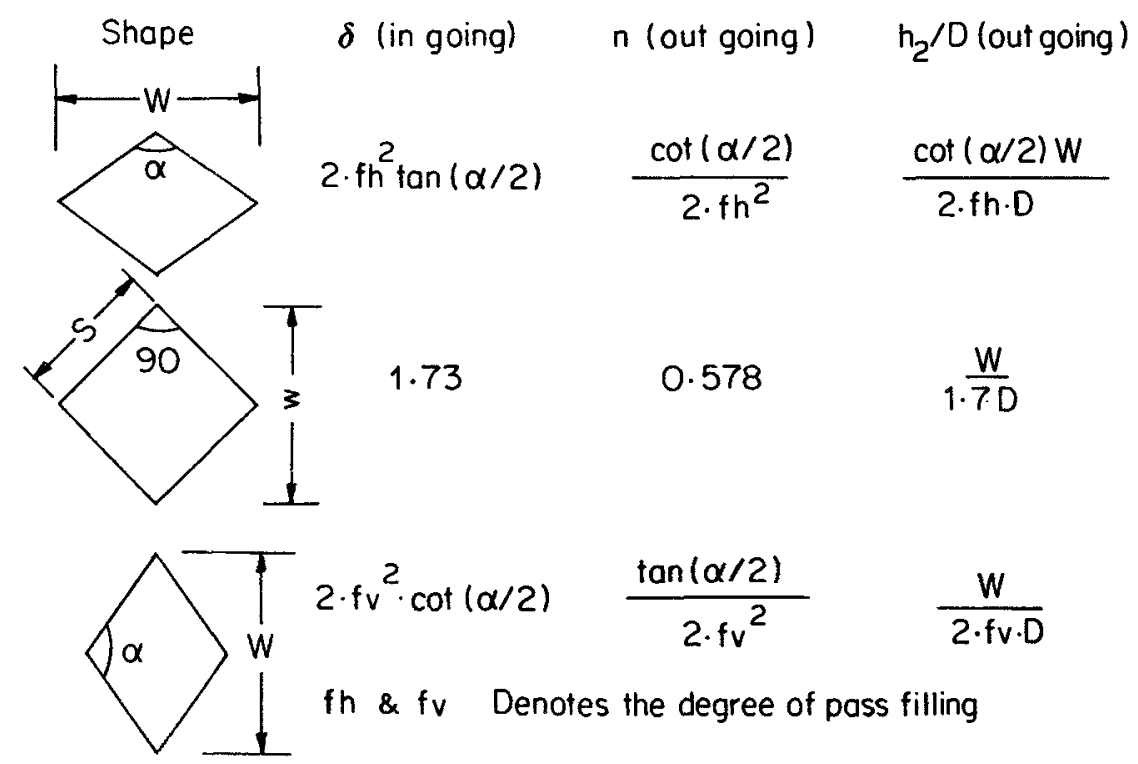

Figure 2. Shape and size factors for diamond and square passes. 
Here

$$
\begin{aligned}
& n_{\sigma}=\frac{\frac{l d}{W}\left[\frac{1-H_{1} / 4 R}{\left(H_{0}+H_{1}\right) / W}+\frac{W}{8 R} \frac{\left(H_{0}+H_{1}\right)}{\left(H_{0}-H_{1}\right)}\right]}{2 / 3-H_{1} / 10 R} \\
& \sigma=\frac{K \cdot \varepsilon^{0.6235} \dot{\varepsilon}^{0.087}}{e^{0.00372}}
\end{aligned}
$$

\subsection{Rolling torque}

$$
\begin{aligned}
& T=2 \cdot \sigma \cdot \psi \cdot l d^{2} \cdot W\left[M_{1}\left(0.5-\frac{H_{1}}{8 R}\right)+\frac{0.25}{M_{1}}\right] \\
& M_{1}=l d / H_{m} \\
& l d=\left[R\left(H_{0}-H_{1}\right)\right]^{1 / 2}
\end{aligned}
$$

The terms used in the above mathematical expressions are explained in the list of symbols given at the end of the paper.

\section{Mathematical model}

A mathematical model has been developed for diamond square sequence using the shape and size factor of the pass along with elongation and spread equations. The flow charts of the computer program developed for the above model are shown in figures 3a, b. Figure 3a shows the flow chart of the program taking the limitation of initial square size and optimizing the apex angle. Figure $3 \mathrm{~b}$ shows how elongation coefficient was optimized at all mill stands by considering the limitation of power force parameter and biting ability etc and compute the geometrical dimensions of the passes. The program (figure 3a) computes the shape and size factor from the initial assumed value of the diamond angle. Using the shape and size factor, the reduction $(\gamma)$ was computed from the spread equation given by (2). The spread equation has been solved by employing Newton's substitution iterative method. For this we start with an arbitrary value of $\gamma$ in the first iteration and a new value of $\gamma$ is obtained. The process is repeated until convergence (accuracy: $10^{-3}$ ) is achieved, normally after 6 iterations. After getting the value of $\gamma$ and taking the finish square size as the starting dimensions, the elongation, bite angle etc were computed using the corresponding subroutine. The process was repeated till the required pass. If the square size so obtained after the last pass is less than the required initial size, the apex angle of last diamond pass is increased by $2^{\circ}$ and the initial square size is again computed. If again the computed square size is less than the required size of square, the apex angle of pre-last and subsequent diamond passes are increased by $2^{\circ}$ and the square size is computed.

The process is repeated until the calculated square size is greater than the required square size. If the apex angle is given for all the diamond passes then starting with the finish size the program will compute the required initial square size for that finish square size. It is essential to have the following initial data for operation of the computer model: (a) mode of pass sequence; (b) no. of passes; (c) roll diameter at each stand; (d) roll gap at each stand; (e) final rolling speed; (f) final product size; (g) initial 
size and (h) apex angle of all the diamond passes. Once this is computed it can be compared with the actual mill parameter.

\section{Results and discussion}

To ascertain the accuracy of the model developed, a case study was made. In this, geometrical dimensions of square-diamond-square pass sequence for a known 4-pass

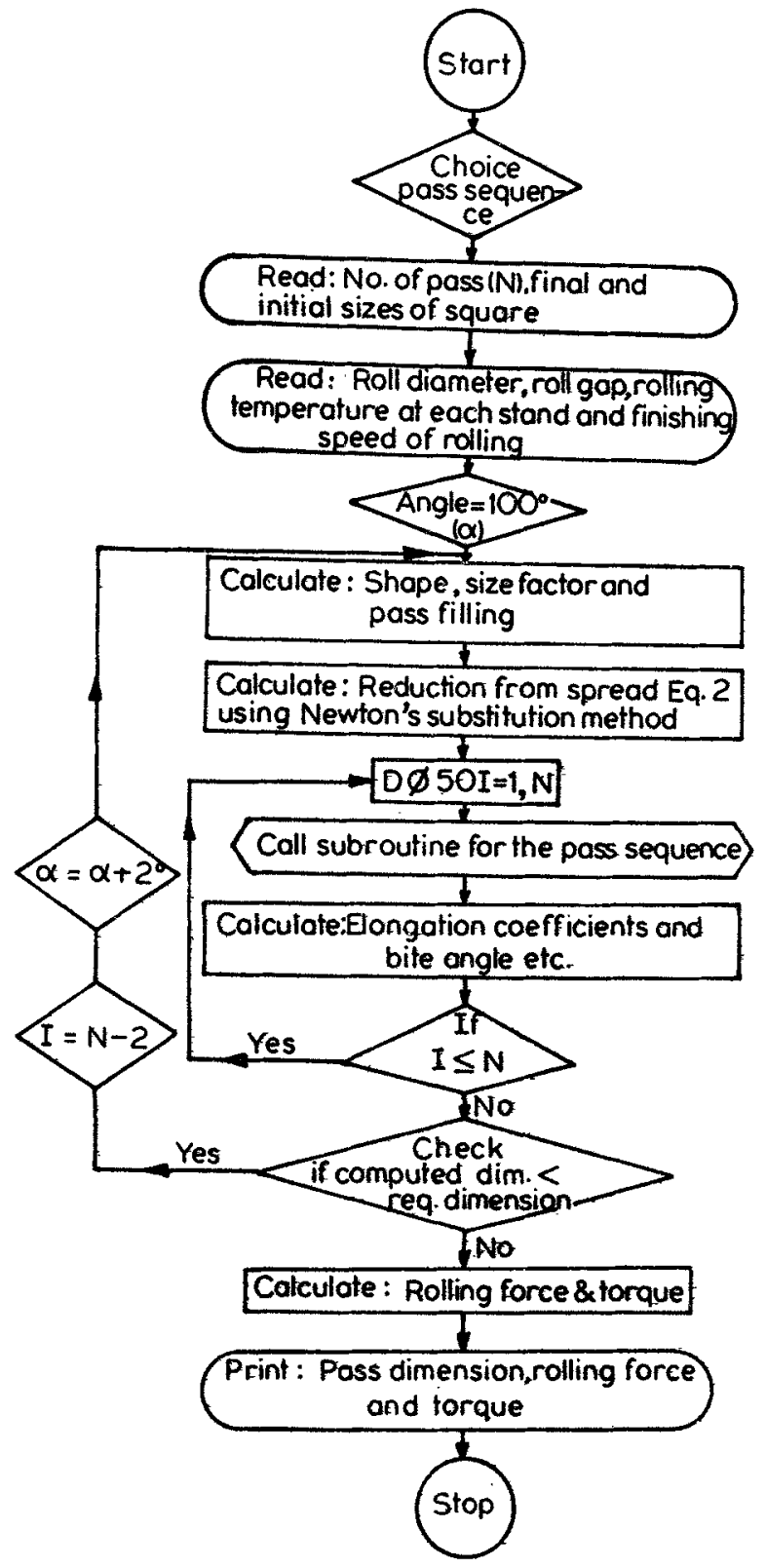

Figure 3a. for caption, see p. 188. 


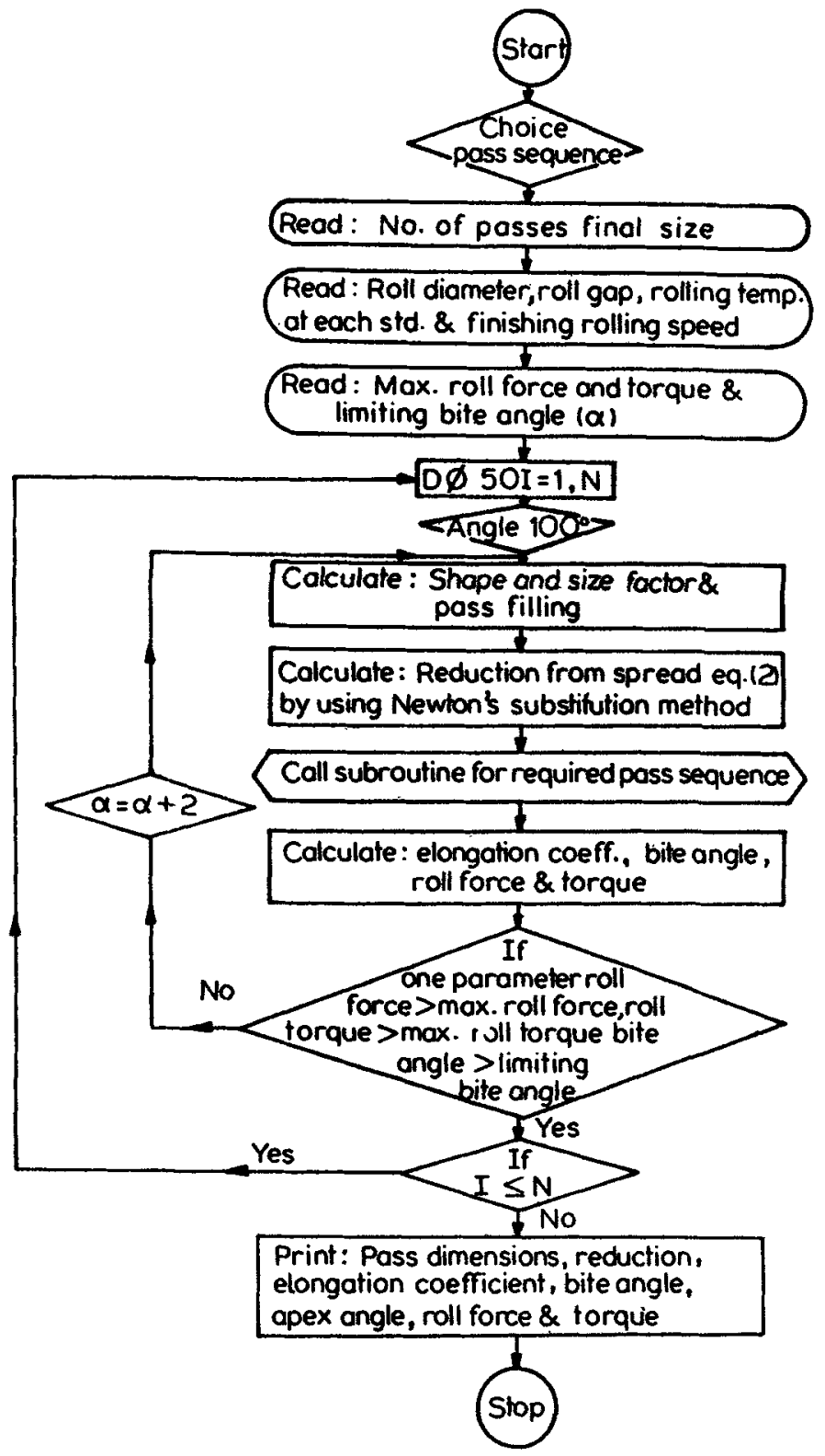

(b)

Figure 3. Flow chart of computer program for calculating roll passes with limitations of a. initial square size. b. Power force and biting angle.

sequence (Bakhtinov and Shternov 1953) are computed using the above model by taking the final square size of $99.7 \mathrm{~mm}$ as the starting dimensions. The roll diameter, roll gap and apex angle of diamond used for calculating the geometrical dimensions are given in the table 1 along with the elongation value and reduction in each pass. The 


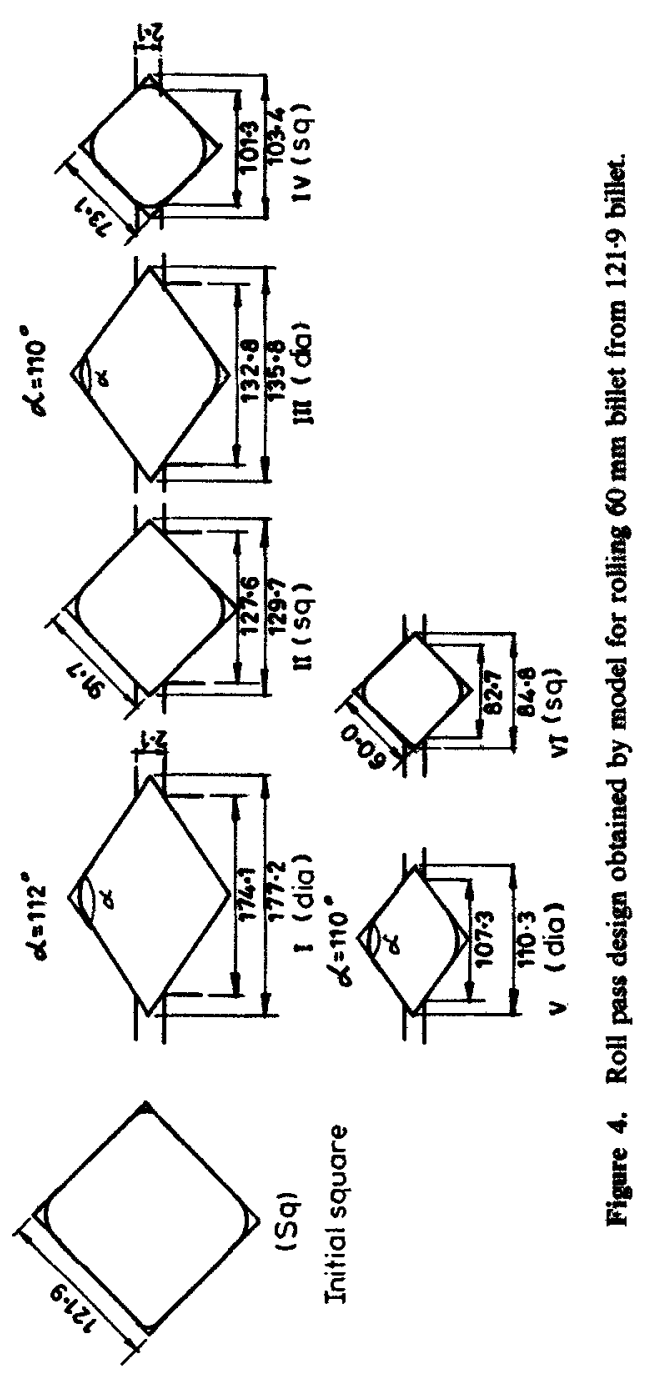


calculated square and diamond sizes are compared with the actual data obtained from the mill. This is also shown in table 1. A comparison between these two values shows a close agreement. Discrepancy in the width of the diamond pass may be due to the extent of pass filling and pass rounding which was considered in the above model.

\subsection{Roll pass design}

A model has been used to design roll passes for the finishing train of a continuous $530 \mathrm{~mm}$ billet mill producing $60 \mathrm{~mm}$ square billet from input square of $120 \mathrm{~mm}$ in six passes. The results are shown in figure 4 with geometrical dimensions of passes. The apex angle of diamond passes has been manipulated in the model to achieve the desired input square. As can be seen in figure 5, with increasing apex angle of diamond, the elongation coefficient increases. With diamond angle of $110^{\circ}$ in all the passes, the initial square size permitted according to model is $119.25 \mathrm{~mm}$. This necessitates increasing the apex diamond angle to $112^{\circ}$ in the first pass. It can also be observed from figure 5 that

Table 1. Comparison between computed and in practice roll pass design (starting size of square(s) $99.7 \mathrm{~mm}$ )

\begin{tabular}{|c|c|c|c|c|c|c|c|c|}
\hline \multirow{2}{*}{$\begin{array}{l}\text { Pass } \\
\text { No. }\end{array}$} & \multirow[b]{2}{*}{ Shape } & \multirow{2}{*}{$\begin{array}{c}\text { Roll } \\
\text { dia } \\
\text { (d) (mm) }\end{array}$} & \multirow{2}{*}{$\begin{array}{l}\text { Roll } \\
\text { gap } \\
(\mathrm{mm})\end{array}$} & \multirow{2}{*}{$\begin{array}{c}\text { Apex } \\
\text { angle } \\
(\alpha)\end{array}$} & \multirow{2}{*}{$\begin{array}{c}\text { Elongation } \\
\text { coefficient } \\
\text { (ג) }\end{array}$} & \multirow{2}{*}{$\begin{array}{l}\text { Reduction } \\
\text { (R) }\end{array}$} & \multicolumn{2}{|c|}{$\begin{array}{c}\text { Geometrical } \\
\text { pass dimension }\end{array}$} \\
\hline & & & & & & & Computed & In practice \\
\hline 1 & Diamond & 800 & 10 & 108 & $1 \cdot 19$ & 0.78 & $W=177 \cdot 0$ & $W=169 \cdot 0$ \\
\hline 2 & Square & 800 & 10 & 108 & $1 \cdot 23$ & 0.77 & $S=118 \cdot 1$ & $S=119.5$ \\
\hline 3 & Diamond & 780 & 10 & $111-2$ & $1 \cdot 232$ & 0.75 & $W=223.9$ & $W=229.0$ \\
\hline 4 & Square & 780 & 10 & 111.2 & 1.32 & 0.72 & $S=150-4$ & $S=1500$ \\
\hline
\end{tabular}

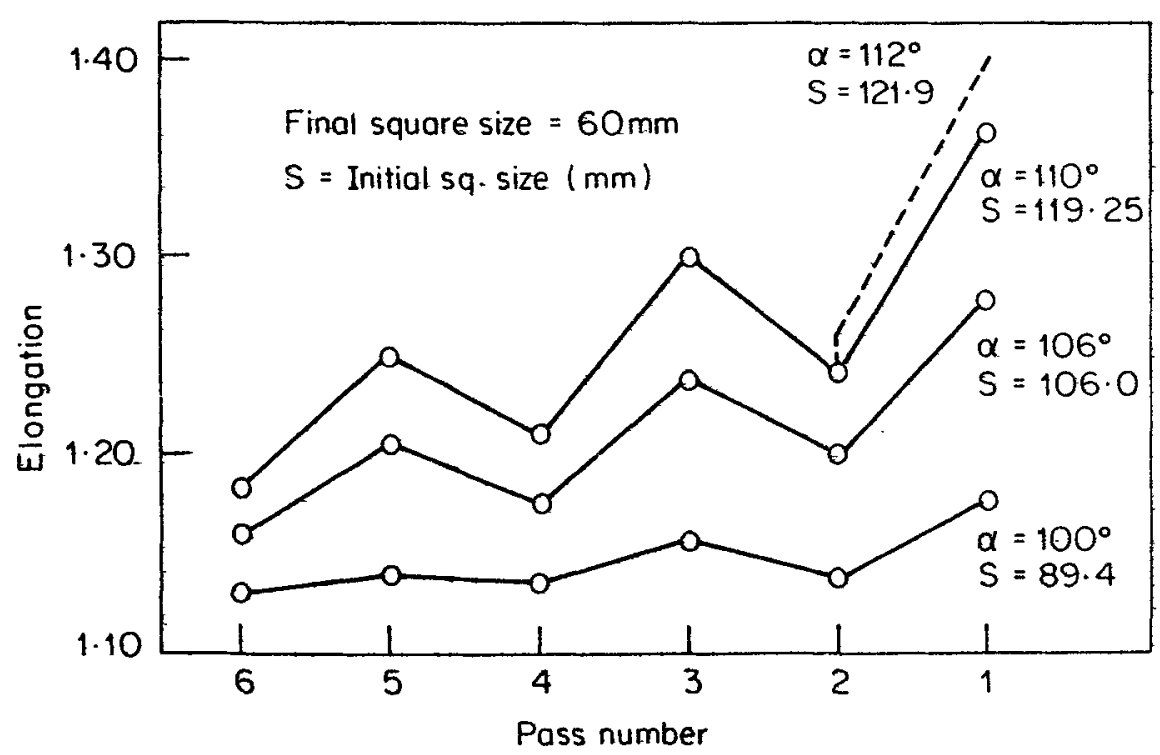

Figure 5. Effect of apex angle on elongation for different passes. 
elongation in square passes is higher compared to those of diamond passes. Nonuniformity of elongation in diamond and square pass increases with increase in apex angle of diamond.

Pass design developed above is being implemented in one of our continuous billet mills.

\section{Conclusions}

A computer-aided roll pass design has been developed for producing billet. The software package accurately calculates the geometrical dimensions of diamond and square passes either under the limitation of rolling force, rolling torque, biting angle etc or the input bloom size for different mill stands thus avoiding trial and error method normally practised by pass designers. The model has been verified with actual plant data and it is shown that the calculated and observed size of the passes is in close agreement.

\section{References}

Bakhtinov and Shternov 1953 Roll pass design (Moscow: Metallurgia) p. 232

Burtsev K 1973 Rolling practice (Moscow: Mir Publishers) p. 146

Jha S 1980 Steel India 31

Jha S, Acharya N K and Sengupta D 1983 Investigation into rolling technology of continuous billet mill, Internal Report, Research and Development Centre for Iron and Steel, Ranchi, 16.02.480.85

Zaitsev M L and Anufriev G V 1969 Stal in English, 8x Vol. 8, p. 727

\section{List of symbols}

D working diameter of outgoing pass

$\boldsymbol{R} \quad$ collar diameter of rolls

$F$ rolling force

$S \quad$ square size (outside)

$H_{0}, H_{1}$ initial and final height of pass

$t \quad$ rolling temperature

$H_{m} \quad$ average height

$h_{2} / D$ size factor of pass

$T$ rolling torque

$K$ constant

ld arc of contact

$W \quad$ width of diamond pass

$\gamma \quad$ reduction at pass

$\delta, n \quad$ shape factor of passes

$M \quad \frac{0.8}{\left(b_{1} / l d\right)^{2}}\left[2-\frac{3\left(h_{1}-h_{2}\right)}{l d}\right]$

$\varepsilon, \dot{\varepsilon} \quad$ strain and strain rate

$n_{\sigma} \quad$ stress coefficient

$\lambda$ elongation coefficient

$\sigma \quad$ yield stress

$\psi \quad$ lever arm ratio 